\title{
Remote Sensing using Bistatic GPS and a Digital Beam-Steering Receiver
}

\author{
Alison K. Brown, Ph.D. \\ NAVSYS Corporation \\ Colorado Springs, CO, USA \\ abrown@navsys.com
}

\begin{abstract}
GPS bistatic signals have applications for remote sensing in collecting data such as soil moisture content, surface altitude or wave speed. Prior research using these signals has been limited by the low signal power of the bistatic GPS signals. NAVSYS Corporation has developed an advanced bistatic GPS receiver that uses a 109-element GPS antenna array and digital beam steering to provide gain to increase the ability to detect the weak bistatic GPS signals. This provides up to $20 \mathrm{~dB}$ of gain over previous receivers.
\end{abstract}

In this paper, the design of the Digital Beam-Steering receiver is described and data collected during flight tests with the array are presented. The data was collected with the antenna array installed on a Cessna aircraft. Flights were conducted over terrain and water and the data was recorded for post-test analysis. The results of the flight test show the increase in fidelity and observability of the bistatic GPS signals by using digital beam steering. The digital scanning capability of the receiver also increases the area of coverage over which data can be collected from a single aircraft pass. The enhanced data collected will be of benefit for all remote sensing applications using bistatic GPS signals.

\section{INTRODUCTION}

Early experimentation using NAVSYS' advanced Global Positioning System (GPS) receiver technology, demonstrated the ability to track the reflected GPS signals from the surface of the earth in the early 90's [1]. Since then, further research has demonstrated the utility of these signals for applications such as surface altimetry [2], wave motion detection and wind sensing [3], and observing surface water content [4,5] for mapping ice fields or wetlands.

Because of the extremely low power level of the returned bistatic GPS signals, this previous research has focused primarily on the strong specular bistatic signals. NAVSYS has developed a Digital Beam-Steering GPS receiver, the Highgain Advanced GPS Receiver (HAGR), which can be used to increase the received signal/noise ratio from these weak bistatic signal returns allowing improved detection of both specular and diffuse GPS signals (Figure 1). The theoretical basis for the GPS bistatic sensing using these signal returns is included in Reference [6].

\section{DigitAL BEAM-STEERING GPS RECEIVER}

The NAVSYS High-gain Advanced GPS Receiver is a digital beam steering receiver designed for GPS satellite radio navigation and other spread spectrum applications. This is installed in a rugged Compact PCI chassis (Figure 2) suitable for aircraft flight tests.

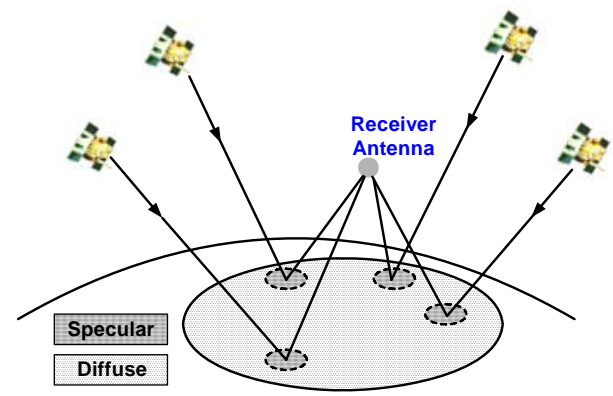

Figure 1 GPS Bistatic Geometry with Specular Reflection Points

The HAGR system architecture is shown in Figure 3. The signal from each antenna element is first digitized using a Digital Front-End (DFE). Each DFE card includes the capability to sample signals from 8 antenna inputs. These can be cascaded together to allow beam steering to be performed from a larger antenna array. The complete set of DFE digital signals is then used to create the composite digital beamsteered signal input by applying a complex weight to combine the antenna array outputs. The HAGR can be configured with a variable number of antenna elements up to a total of 109elements, as shown in Figure 4. For the first flight test a 15element array was used, with the elements shown in red in Figure 4. Figure 5 and Figure 6 shows the 15-element and 109-element beam pattern created by this array. Through the HAGR digital control, these beams can be directed at any point on the surface of the earth for data collection. The area they cover is a function of the beam width and the aircraft altitude, as illustrated in Figure 7. Up to 5 beams each, with $+20 \mathrm{~dB}$ gain, can be independently directed by the HAGR signal processing.

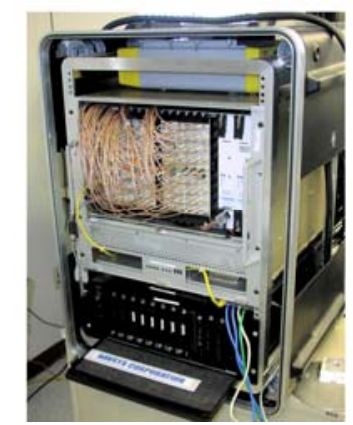

Figure 2 HAGR Assembly 


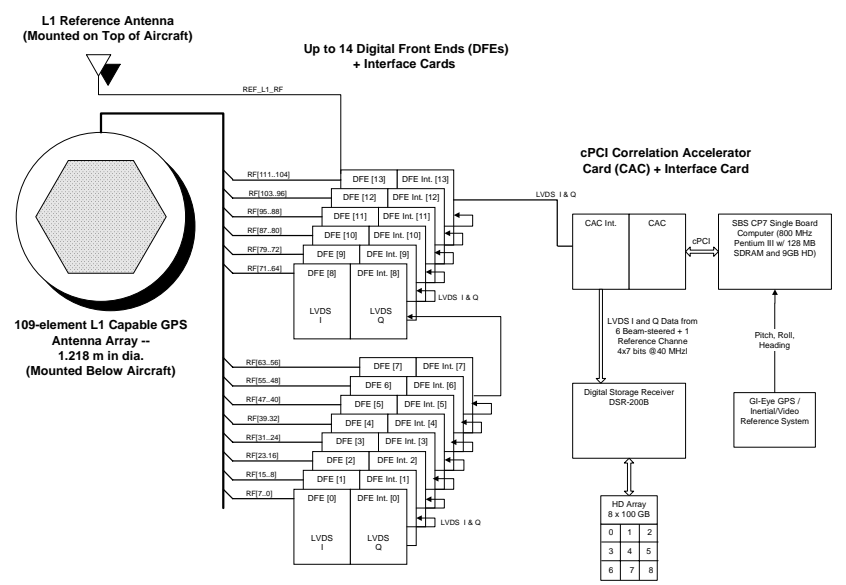

Figure 3 HAGR System Architecture

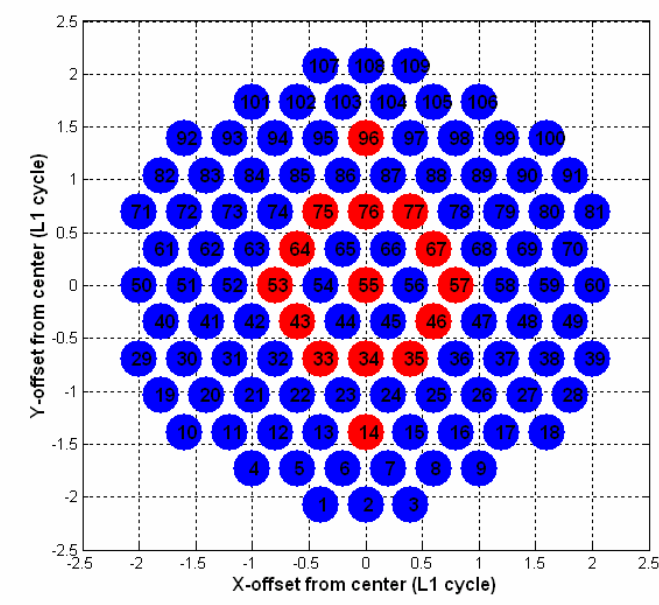

Figure 415 and 109-Element Phased Array

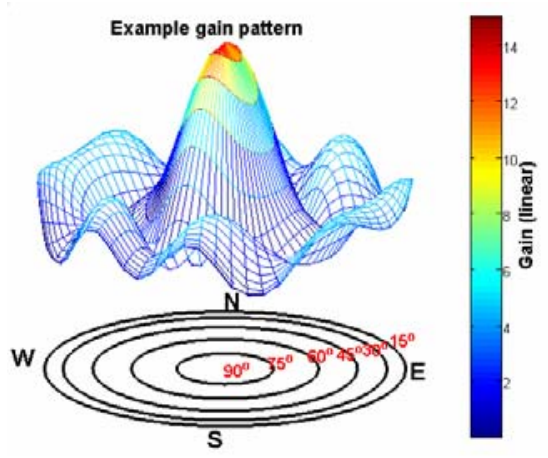

Figure 5 Beam Pattern of 15-element Phased Array

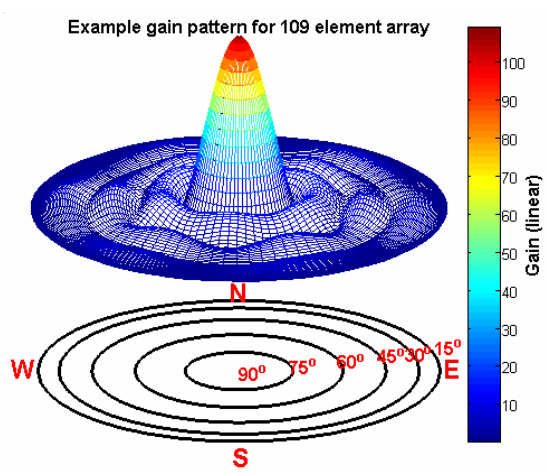

Figure 6 Beam Pattern of 109-Element Array

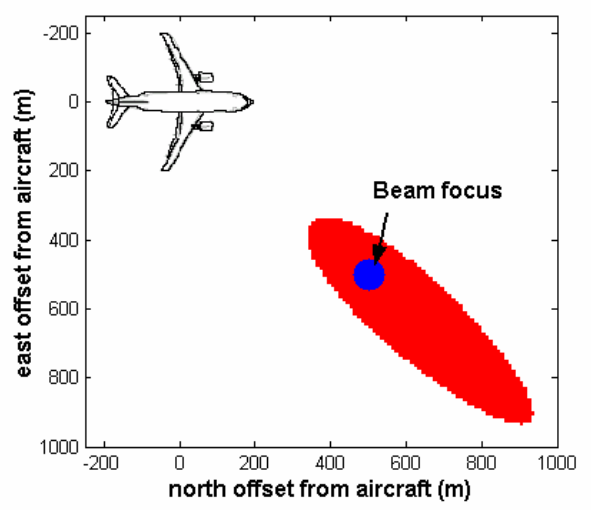

Figure 7 109-Element Beam Footprint (3dB contour from 500 m altitude)

\section{BistatiC GPS FLIGHT TEST}

The first flight test was conducted with the 15-element HAGR antenna array and digital beam-steering receiver. The antenna array was installed on the underside of a Cessna test aircraft, Figure 8, and a reference antenna was installed on the upper-side of the aircraft. During this flight test, the HAGR was used to track the GPS satellites and the raw broad-band data was also recorded from each of these elements, and a reference antenna using our digital storage receiver $[7,8]$. Approximately one hour of bistatic maritime data and two hours of bistatic land data was collected. This data was then played back into the HAGR from the digital storage receiver for signal processing post-test.

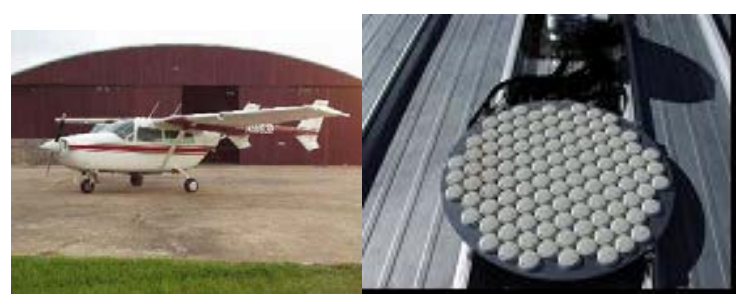

Figure 8 Cessna Test Aircraft and Antenna Array

\section{SPECULAR DATA ANALYSIS}

Analysis of the specular return over land can be used to provide information on both elevation [2] and also the landtype where the signal is being returned (e.g. water content) [4, 
5]. For example, Figure 9 shows a dramatic increase in return power when the specular point crosses the Pearl River in a forest on the Mississippi-Louisiana border. The rough surface formed by the treetops provides a low specular return whereas the smooth river surface provides a very strong return.
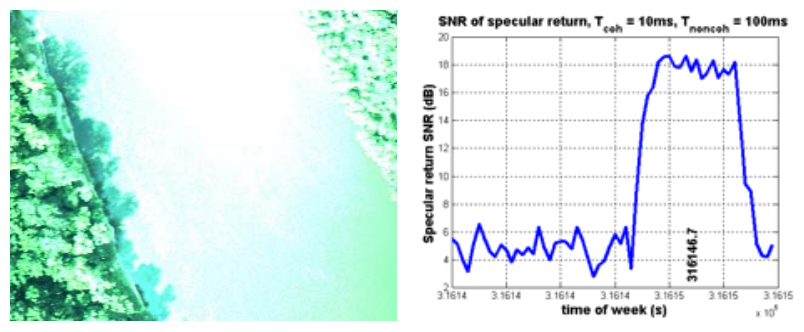

Figure 9 Pearl River Crossing, Specular Power Increase

\section{MARITIME DIFFUSE DATA ANALYSIS}

Over water, there is a very strong specular return and a very weak diffuse signal return. To bistatic GPS data was analyzed over water to determine whether it could be used to detect signals of interest from the diffuse bistatic signal returns from vessels on the surface of the water. The recorded GPS data was analyzed over a surface area containing the stationary oil tanker shown in Figure 11. As shown in Figure 11, this target was at some distance from the specular regions of the GPS signal returns. The post-processed bistatic signal returns from the satellite signals processed are shown in scenario. The results shown in Figure 12 indicate a strong return from the tanker's location only from one of the satellite signal, SV 14 only. From examination of Figure 11, this was the only satellite signal that provided a bistatic backscattered return. This indicates that this target was likely detected from backscattered GPS signals returned through a corner reflection type of effect.

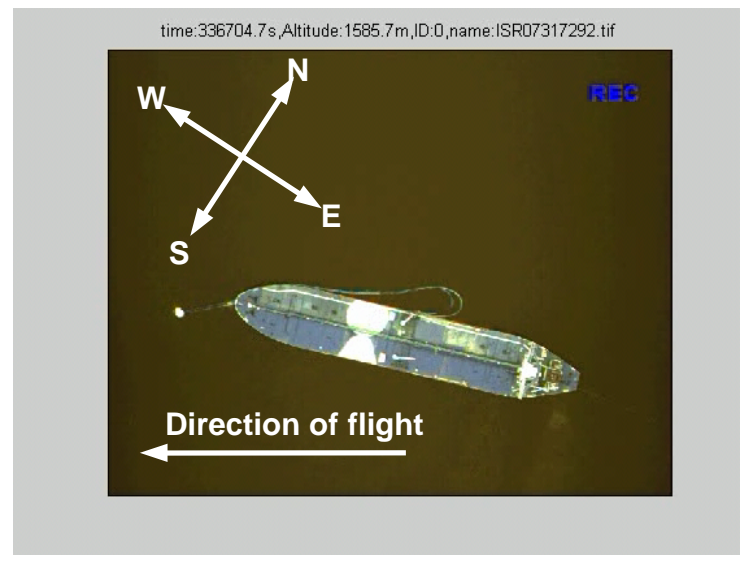

Figure 10 Direction of Flight

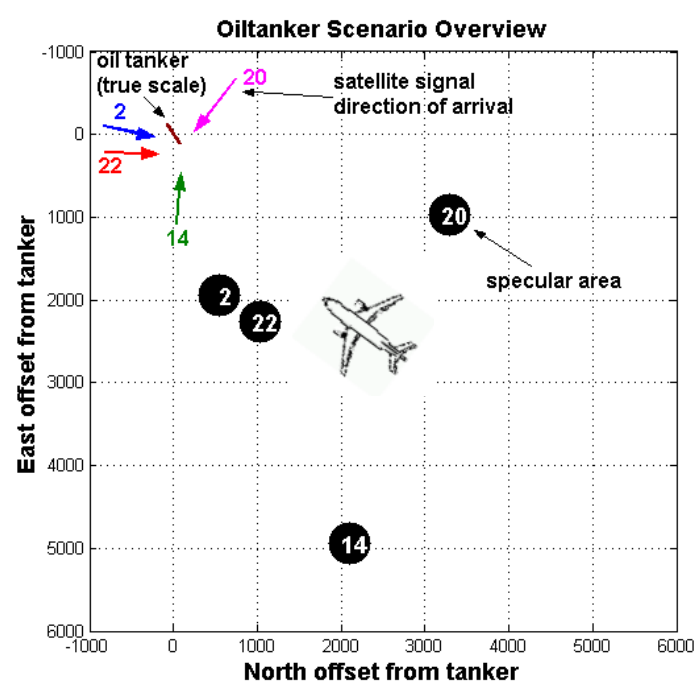

Figure 11 Maritime Bistatic Scenario
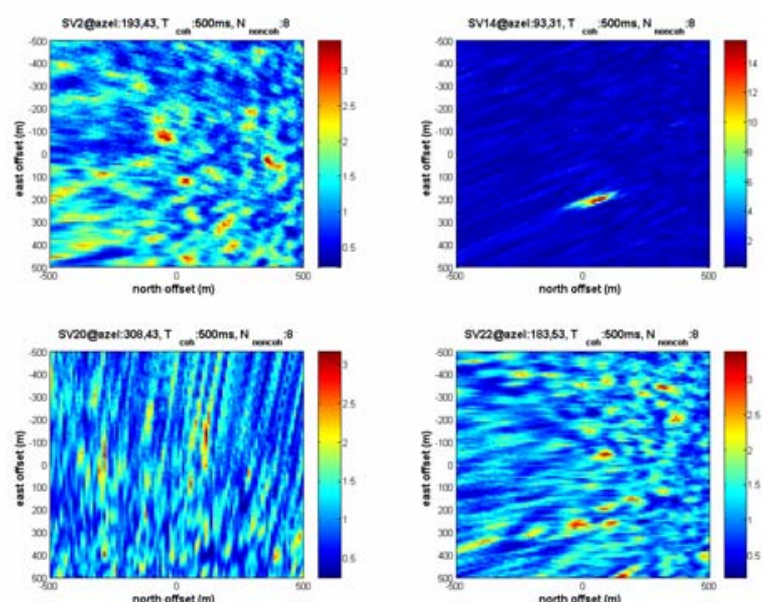

Figure 12 Maritime Bistatic Diffuse Signal Returns (Strong return only for satellite 14)

\section{LAND DIFFUSE DATA ANALYSIS}

The recorded data was also analyzed to evaluate the magnitude of the diffuse bistatic GPS returns over land. This data was also used to build a GPS bistatic signal simulation tool for use in predicting the magnitude of the bistatic signatures as a function of the signal geometry and the modeled land clutter coefficients [6]. Figure 13 and Figure 14 compare the results of the model-based simulation and the actual processed bistatic signal returns from the flight test data. This shows that we have good correspondence between the bistatic simulation model and the real-world data. Because of the low level of the signal returns, reliable bistatic signal detection is only possible with the 15-element array over a small region. The Matlab model-based simulation tool was used to predict the area of coverage over which bistatic GPS signals could be expected to be detected for the 109 element antenna array which will be used in the next scheduled flight test. From Figure 15, this predicts that a much larger region of interest is covered using this larger version of the phased array which will 
open up some interesting new opportunities for remote sensing applications. This will be explored in the flight testing being conducted in 2005.

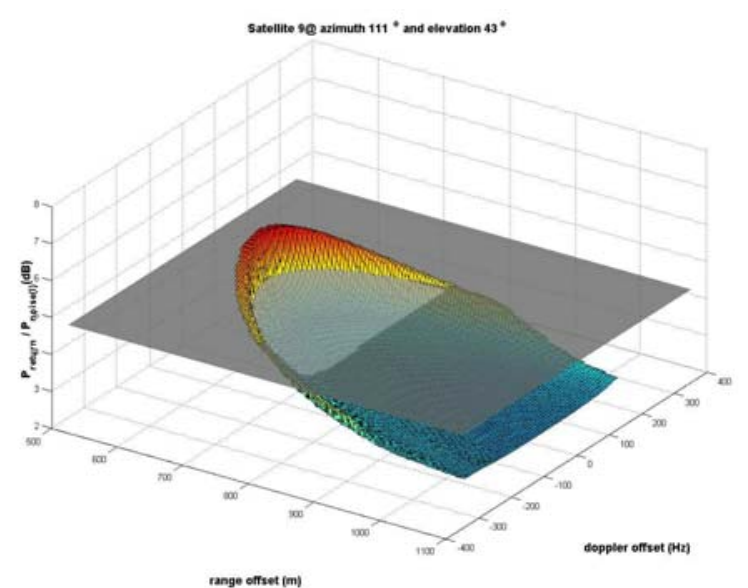

Figure 13 Modeled Bistatic Signals SV 9 (SNR Peak=7 dB)

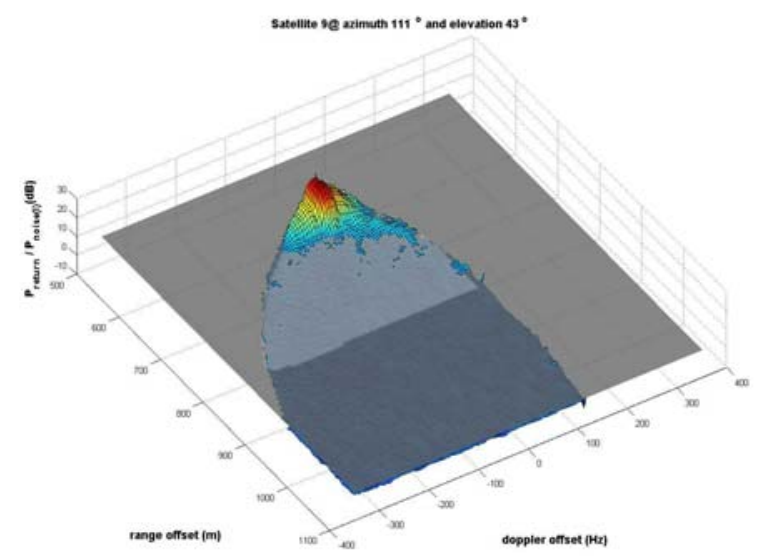

Figure 14 Actual Bistatic Signals SV 9 (SNR Peak=24 dB)

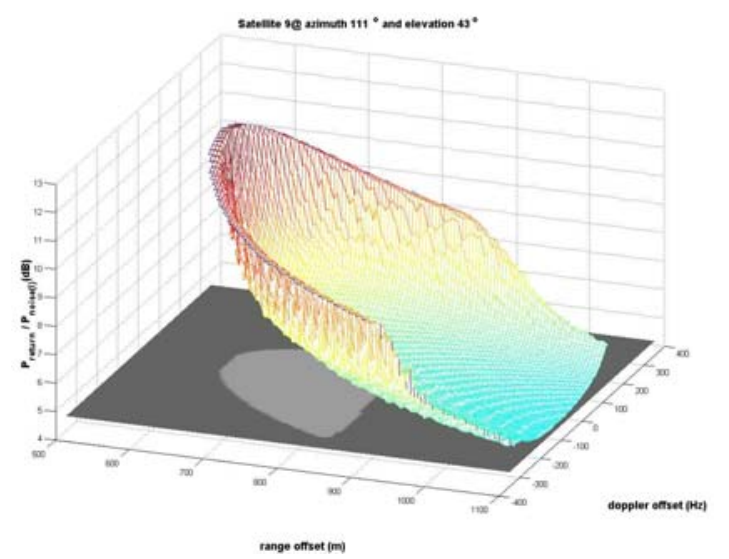

Figure 15 Simulation for 109 element array (SV 9)

\section{CONCLUSION}

The test results and analysis described in this paper have demonstrated the ability of the HAGR receiver to improve the GPS bistatic remote sensing capability by using a digital beam steered to allow the weak bistatic GPS signal returns to be detected over a larger area. The flight test results were used to demonstrate the following performance improvements possible with this receiver design.

- Robust detection and tracking of specular signals over both land and water

- Detection of diffuse signal returns over water from some surface vessels where backscatter occurred

- Detection of diffuse signal returns over a region of interest over land

Flight testing of the 109 element phased array is expected to demonstrate further performance improvements and the ability to process both the specular and the diffuse GPS satellites signal returns, increasing the utility of this sensor for remote sensing applications.

\section{ACKNOWLEDGEMENTS}

The authors would like to acknowledge the support of Charles Luther of Office of Naval Research for sponsoring this activity. This work was funded under SBIR Contract No. N00014-00-C-0552.

\section{REFERENCES}

[1] J. Auber at al, "Characterization of Multipath on Land and Sea at GPS Frequencies", Proceedings of the Institute of Navigation GPS-94 Conference, Salt Lake City, Utah [2] D. Masters, P. Axelrad, V. Zavorotny, S.J. Katzberg, and F. Lalezari, “A Passive GPS Bistatic Radar Altimeter for Aircraft Navigation,” ION GPS-2001, Salt Lake City, OR, p. 2435-2445, September 2001

[3] V.U. Zavorotny, "Bistatic GPS Signal Scattering from an Ocean Surface: Theoretical Modeling and Wind Speed Retrieval from Aircraft Measurements," Workshop on Meteorological and Oceanographic Applications of GNSS Surface Reflections: from Modeling to User Requirements, July 6, 1999, De Bilt, The Netherlands, http://www.etl.noaa.gov/ vzavorotny/

[4] J. Garrison, S. Katzberg, "The application of reflected GPS signals to ocean and wetland remote sensing,” In the Proceedings of the Fifth International Conference on Remote Sensing for Marine and Coastal Environments, San Diego, CA, 5-7 October, Vol. 1, pp. 522-529, 1998.

[5] D. Masters, V. Zavorotny, S. Katzberg, and W. Emery, "GPS Signal Scattering from Land for Moisture Content Determination”. Presented at IGARSS, July 24-28, 2000. http://ccar.colorado.edu/ dmr/pubs/papers/masters2000igarss soil_doc.pdf

[6] K. Stolk and A. Brown, "Bistatic Sensing with Reflected GPS Signals Observed with a Digital Beam-Steered Antenna Array,” Proceedings of ION GPS 2003, Portland, Oregon, September 2003

[7] A. Brown and N. Gerein, "Advanced GPS Hybrid Simulator Architecture”, Proceedings of ION 57th Annual Meeting 2001, Albuquerque, NM, June 2001

[8] http://www.navsys.com/Products/aghs.htm 